\title{
Design and development of anchoring positioning control system for riprap leveling ship
}

\author{
Xiaotao Hua ${ }^{1,2,3,4}$, Yan $\operatorname{Liu}^{5 *}$, Haiyang Sun ${ }^{5}$ and Jianru Chen ${ }^{5}$ \\ ${ }^{1}$ CCCC Second Harbor Engineering Company Ltd, 430040, China \\ ${ }^{2}$ Key Laboratory of Large-span Bridge Construction Technology, 430040, China \\ ${ }^{3}$ Research and Development Center of Transport Industry of Intelligent Manufacturing Technologies of Transport Infrastructure, 430040, \\ China \\ ${ }^{4}$ CCCC Highway Bridge National Engineering Research Centre Co. Ltd, 430040, China \\ ${ }^{5}$ Huazhong University of Science and Technology, School of Naval Architecture and Ocean Engineering, 430074, China
}

\begin{abstract}
It is very important to level foundation bed by riprap in water and soil engineering. In this paper, a real-time feedback convergence control method is proposed to control the position and heading angle of the riprap leveling ship. The wind, wave, current and hydrodynamic parameters are obtained by empirical formula; the tension of four cables is calculated according to the balance equation of six degrees of freedom, and then the cable deformation is obtained. According to the deformation of the cable, the length of the cable in a certain equilibrium state can be obtained. The length of four cables can be lengthened or shortened by comparing the length of cables at two balanced positions. The length of cables can be controlled by winch to complete the anchoring and positioning control of leveling ship.
\end{abstract}

\section{Introduction}

The riprap leveling ship is a kind of anchoring and shifting ship (non self-propelled ship). During the construction, the course and track of the ship can be maintained or changed by coordinating and controlling the action and speed of multiple windlass. Among the existing positioning and moving ships in shallow water, mooring system is the most widely used. In comparison, the research on dynamic positioning system is relatively mature at home and abroad ${ }^{[1]}$, but for the mooring positioning system of non self propelled ships, the research results are still less in terms of motion modeling and controller design. With the increasing application of engineering ships, the theoretical research of mooring positioning system will be more and more, such as the combination of dynamic positioning and dynamic positioning to achieve active positioning and improve positioning accuracy[2-5].

The east-west axis road project of Xiangyang City is a major traffic channel connecting Fancheng with the central area of Dongjin new city. The tunnel passes through Yuliangzhou to protect the ecological landscape of Yuliangzhou. The total length of Yuliangzhou tunnel in Xiangyang east-west axis road project is $5400 \mathrm{~m}$. The immersed tube scheme is adopted to cross the Hanjiang river twice, with a total length of $1011 \mathrm{~m}$. The project requires that the horizontal positioning accuracy of gravel laying and leveling of subgrade bed shall not exceed $20 \mathrm{~cm}$, and the single displacement time shall not exceed 10min. Due to the overall size, draft and other factors, the traditional large-scale construction vessels can not enter the narrow operation area of inland river. Secondly, the immersed tube distance of immersed tunnel in inland river basin is generally short, the total immersed tube distance of the project is $1011 \mathrm{~m}$, and the cost of traditional operation equipment design and procurement is high. From the perspective of cost, construction quality, construction period and feasibility of immersed tube tunnel foundation cushion, the technical scheme of laying and leveling gravel for foundation bed of immersed tube tunnel is selected. The application of the first laying method in inland river basin puts forward the research requirements for the overall plan, positioning strategy, ship moving strategy and leveling technology of the leveling ship.

\section{Mooring positioning control system of leveling ship}

\section{1 system composition}

The hardware of the mooring positioning system of leveling ship is composed of computer, PLC control system, RTK GPS satellite positioning instrument, network switch and interface module, 4 winches and driving mechanism (including frequency converter, encoder and cable tensiometer, etc.) and operation console. The control hardware interface adopts RS485 and the communication protocol adopts Modbus RTU.

* Corresponding author: liuyanhust@hust.edu.cn 


\subsection{Control flow}

According to the construction requirements, the starting point and working route of the leveling ship need to be planned on the computer before work, as shown in Figure 1. After the leveling ship enters the predetermined construction position, the pre tightening force is applied to fix the ship. When the construction is completed at a certain position, it needs to reach the next position by cable traction. The position and angle of the leveling ship should be kept basically unchanged during the construction. Therefore, it is necessary to change the tension force on the basis of the pre tightening force to suppress the interference of wind, waves and currents on the ship, and compensate for the change of the ship's attitude caused by the change of the ship's walking and the position of the chute, so that the ship's position and heading angle fluctuate within the scope of not affecting the operation.

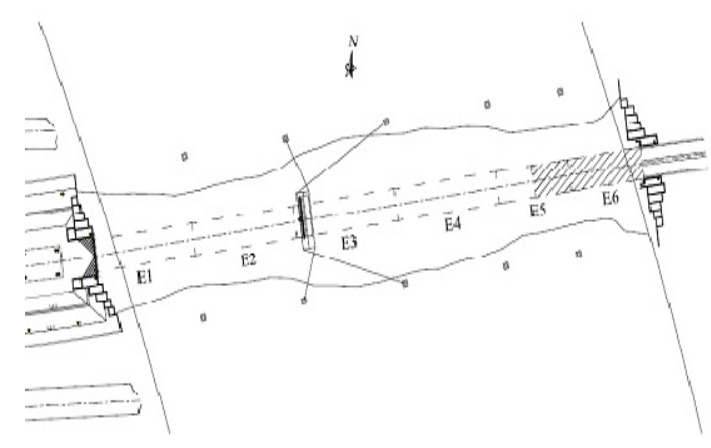

Fig. 1. Operation area of riprap leveling ship.

The control process is generally divided into two steps. The first step is to control the horizontal position of the ship and the ship's heading angle; the second step is the tension compensation in the ship construction stage, in which the cable length is basically unchanged, and the tension is fine-tuning controlled.

\section{Mathematical model}

\subsection{Force analysis of leveling ship}

The external forces during the construction of the leveling ship include gravity, buoyancy, cable tension, wind, wave and current forces, as shown in Figure 2. Because the cable tension is large and the weight per unit length of the selected cable is small, the cable is regarded as a straight line.

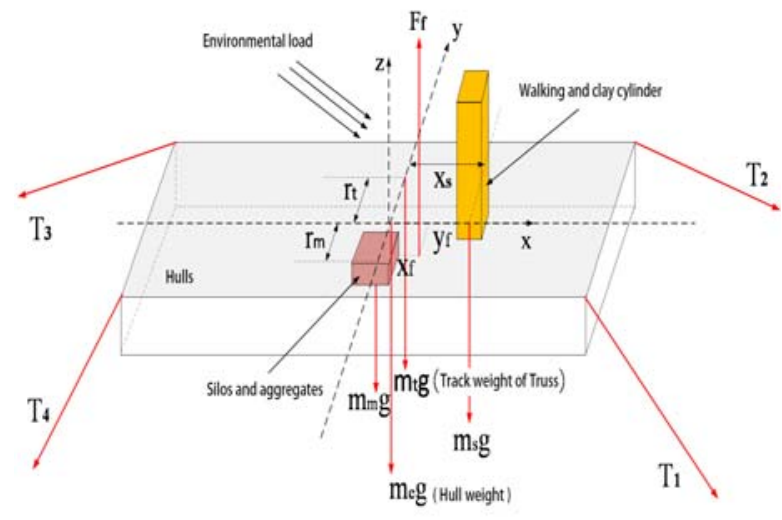

Fig. 2. Force analysis of leveling ship.

Place If we want to keep the leveling ship still during construction, we need to satisfy the balance equation of six degrees of freedom.

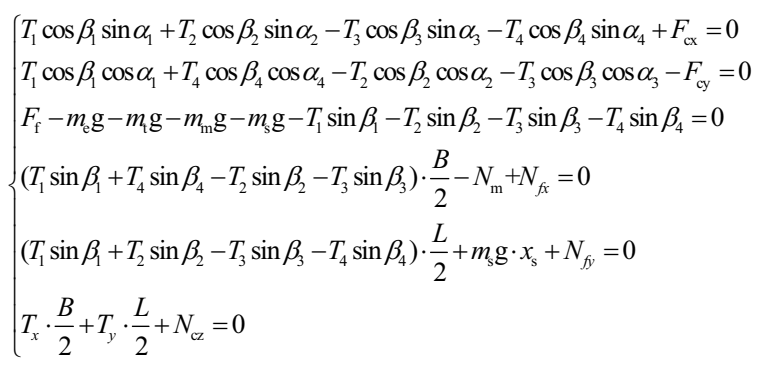

In Eq.1, $T_{1}-T_{4}$ is the tension of four cables; $\alpha_{1}-\alpha_{4}$ is the angle between the projection of four cables in $\mathrm{X}-\mathrm{Y}$ plane and $\mathrm{Y}$ axis (acute angle); $\beta_{1}-\beta_{4}$ is the angle between four cables and $\mathrm{Z}$ axis; $F_{\mathrm{cx}}, F_{\mathrm{cy}}$ and $N_{\mathrm{cz}}$ is the component of environmental load in $\mathrm{X}$ and $\mathrm{Y}$ axis and the moment around $\mathrm{Z}$ axis respectively; $F_{\mathrm{f}}$ is the buoyancy of the ship; $m_{\mathrm{e}}$ is the mass of the empty hull; $m_{\mathrm{t}}$ is the mass of track truss; $m_{\mathrm{m}}$ is the quality of silo and stone; $m_{\mathrm{s}}$ is the weight of walking and chute; $B$ is the width of the ship; $L$ is the length of the ship; $r_{\mathrm{t}}$ is the transverse arm of track truss; $r_{\mathrm{m}}$ is the transverse arm of the silo and stone; $x_{\mathrm{s}}$ and $y_{\mathrm{s}}$ is the longitudinal and transverse arm of walking and sliding drum respectively, which depends on the position of walking and chute; $N_{\mathrm{fx}}$ and $N_{\mathrm{fy}}$ is is the buoyancy moment around x-axis and yaxis respectively; $N_{\mathrm{m}}$ is the heeling moment generated by ship truss, stone mass and traveling chute, as shown in Eq.2.

$$
N_{m}=m_{\mathrm{t}} g \cdot r_{t}+m_{\mathrm{m}} g \cdot r_{m}+m_{\mathrm{s}} g \cdot y_{s}
$$

$\mathrm{T}_{\mathrm{x}}$ and $T_{\mathrm{y}}$ is the resultant force of four cables in $\mathrm{x}-$ axis direction and $\mathrm{Y}$-axis direction respectively, as shown in Eq.3.

$$
\left\{\begin{array}{l}
T_{x}=T_{1} \cos \beta \sin \alpha_{1}+T_{3} \cos \beta_{3} \sin \alpha_{3}-T_{2} \cos \beta_{2} \sin \alpha_{2}-T_{4} \cos \beta_{4} \sin \alpha_{4} \\
T_{y}=T_{1} \cos \beta \cos \alpha_{\mathrm{f}}+T_{3} \cos \beta_{3} \cos \alpha_{3}-T_{2} \cos \beta_{2} \cos \alpha_{2}-T_{4} \cos \beta_{4} \cos \alpha_{4}
\end{array}\right.
$$

For the six degree of freedom equilibrium equation, the tension of four cables can not be solved. Since buoyancy, buoyancy moment around $\mathrm{x}$-axis and buoyancy moment around $\mathrm{Y}$-axis are related to cable tension, they are also regarded as independent variables in this paper. In this way, for a six degree of freedom equation with seven variables, the four cable tensions can be solved by linear optimization, as shown in Eq. 4 . 


$$
\begin{aligned}
& \min f=m\left|N_{f x}\right|+n\left|N_{f y}\right| \\
& \text { s.t. }\left\{\begin{array}{l}
\mathrm{A}_{6 \times 7} \mathrm{~V}_{7 \times 1}=C_{6 \times 1} \\
T_{\max } \geq T_{1} 、 T_{2} 、 T_{3} 、 T_{4} \geq T_{\text {min }}
\end{array}\right.
\end{aligned}
$$

$N_{\mathrm{fx}}$ and $N_{\mathrm{fy}}$ is the buoyancy moment around x-axis and $\mathrm{y}$-axis respectively; $\mathrm{m}$ and $\mathrm{n}$ are the distribution coefficients of heeling and trim respectively; $T_{\min }$ and $T_{\max }$ is the minimum and maximum allowable cable tension respectively.

After the cable tension is obtained by solving the six degree of freedom equation, the total length of the cable can be obtained by coordinate calculation, and then the non deformation length of the cable can be obtained through the calculation formula of cable deformation. This paper assumes that there is a linear relationship between the cable deformation and the tension. When the ship is in position $\mathrm{P}_{1}$, the undeformed length of the four cables is:

$$
l_{i 0}=\frac{l_{i} E A}{T_{i}+E A}(i=1,2,3,4)
$$

In Eq.5, $E$ is the elastic modulus of the cable and $A$ is the cross-sectional area of the cable. At position $\mathrm{P}_{2}$, the undeformed length of the four cables is as follows:

$$
l_{i 0}^{\prime}=\frac{l_{i}^{\prime} E A}{T_{i}^{\prime}+E A}(i=1,2,3,4)
$$

The length of each cable to be lengthened or shortened is as follows:

$$
\Delta l_{i}=l_{i 0}^{\prime}-l_{i 0}(i=1,2,3,4)
$$

\subsection{Influence of preload on ship stability}

After the ship reaches the designated position and starts construction, the cable needs to be preloaded, which has a certain influence on the stability of the ship. In this paper, the influence of preload on the simplified ship model is studied to provide reference for the setting of preload for leveling ship. The motion process of the ship is shown in Fig.3 only considering the environmental force along the $\mathrm{x}$-axis (i.e. the ship centerline). In the figure, $\mathrm{P}_{0}$ is the initial position of the leveling vessel, $\mathrm{P}_{1}$ is the position of the ship after being subjected to environmental forces; $S_{\mathrm{x}}$ is the displacement of ship longitudinal motion; $h_{1}$ is the distance between the connecting point of winch cable at the position $\mathrm{P}_{0}$ and $\mathrm{P}_{1}$.

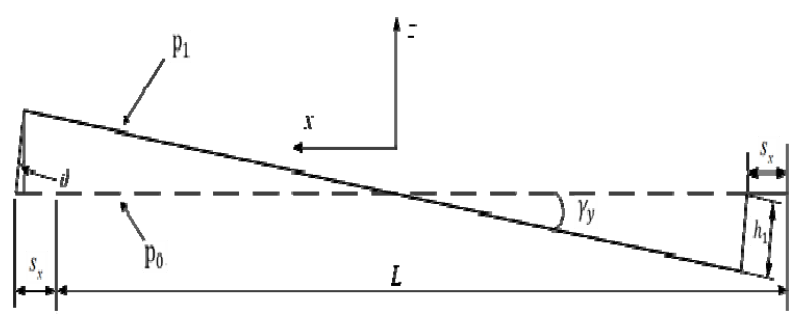

Fig. 3. Schematic diagram of ship's pitch and surge motion.

Due to the small change of buoyancy (less than $0.52 \%$ from the simulation results), the change of ship buoyancy is ignored. At this time, the ship has only two directions of motion, namely yaw and surge. The equation of motion is shown in Eq.8.

$$
\left\{\begin{array}{l}
m \cdot a_{x}=F_{l x}+F_{r x}+T_{1 x}+T_{2 x}-T_{3 x}-T_{4 x} \\
I_{y} \cdot \dot{r}_{y}=N_{f y}+N_{r y}+\left(T_{3 z}+T_{4 z}-T_{1 z}-T_{2 z}\right) \cdot \frac{L}{2}
\end{array}\right.
$$

In Eq.8, $\mathrm{m}$ is the total mass of the ship; $a_{\mathrm{x}}$ is the acceleration along the $\mathrm{X}$ axis; $F_{\mathrm{lx}}$ is the environmental force along the x-axis; $F_{\mathrm{rx}}$ is the resistance of ship motion; $T_{1 \mathrm{x}}-T_{4 \mathrm{x}}$ is the component force/ of tension of four cables in $\mathrm{x}$-axis direction; $l_{\mathrm{y}}$ is the moment of inertia about the y-axis; $r_{\mathrm{y}}$ is the yaw angular acceleration; $N_{\mathrm{fy}}$ is the buoyancy moment around the y-axis; $N_{\mathrm{ry}}$ is the resistance moment of yaw motion; $T_{1 \mathrm{z}}-T_{4 \mathrm{z}}$ is the component force of tension of four cables in z-axis direction.

By integrating the acceleration and angular acceleration, the ship's pitch displacement and yaw angle can be obtained.

$$
\left\{\begin{array}{l}
v_{x}=\int_{0}^{t} a_{x} \mathrm{~d} t, \quad s_{x}=\int_{0}^{t} v_{x} \mathrm{~d} t \\
r_{y}=\int_{0}^{t} \dot{r}_{y} \mathrm{~d} t, \quad \gamma_{y}=\int_{0}^{t} r_{y} \mathrm{~d} t
\end{array}\right.
$$

According to the trigonometric relationship in Fig. 3, it can be concluded that:

$$
\begin{gathered}
\theta=\frac{\pi}{2}-\frac{\pi-\gamma_{y}}{2} \\
h_{1}=\sqrt{2\left(\frac{B}{2}\right)^{2}-2\left(\frac{B}{2}\right)^{2} \cos \gamma_{y}}
\end{gathered}
$$

If the ship is at the center of the four anchor points in the initial state of the ship, the initial length of the four cables is equal. As the ship's attitude changes, the length of the cable also changes. According to the trigonometric relationship and Eq.9-Eq.10, the length of four cables can be obtained.

$$
\left\{\begin{array}{l}
l_{1}=l_{2}=\left(b_{0}-s_{x}+h_{1} \sin \theta\right)^{2}+\left(h_{0}+h_{1} \cos \theta\right)^{2}+c_{0}^{2} \\
l_{3}=l_{4}=\left(b_{0}+s_{x}+h_{1} \sin \theta\right)^{2}+\left(h_{0}-h_{1} \cos \theta\right)^{2}+c_{0}^{2}
\end{array}\right.
$$

In Eq. $12, \mathrm{~b}_{0}$ and $\mathrm{c}_{0}$ are the longitudinal and transverse distances from the anchor point of the ship's winch (or the connection point between the winch and the cable), respectively.

After the total length of the cable is obtained, the cable tension can be obtained according to the cable deformation formula. There is a linear relationship between the cable tension and the cable length if the length of the cable does not change during the loading process:

$$
l_{i}=l_{0} \cdot \frac{T_{i}+E A}{E A}(i=1,2,3,4)
$$

When the ship's attitude is changed, the angle $\beta_{\mathrm{i}}$ between the cable and the $Z$ axis and the angle $\alpha_{i}$ between the projection of the cable in the XY plane and the $\mathrm{Y}$ axis also change. 


$$
\left\{\begin{array}{l}
\alpha_{1}=\alpha_{2}=\arctan \frac{b_{0}-s_{x}}{c_{0}} \\
\alpha_{3}=\alpha_{4}=\arctan \frac{b_{0}+s_{x}}{c_{0}} \\
\beta_{1}=\beta_{2}=\arcsin \frac{h_{0}+a_{1} \cos \theta}{l_{1}} \\
\beta_{3}=\beta_{4}=\arcsin \frac{h_{0}-a_{1} \cos \theta}{l_{1}}
\end{array}\right.
$$

The surge resistance of the ship is proportional to the square of the speed.

$$
F_{r x}=-C_{r x} \cdot v_{x}^{2}
$$

Due to the regular shape of the trim ship, after simplified calculation, the ship's trim buoyancy moment has a linear relationship with the ship's trim angle.

$$
N_{f y}=-C_{f y} \cdot \tan \gamma_{y}
$$

Since the working area of the leveling ship is a river vessel, the influence of waves is small. In order to simplify the calculation, the relationship between the pitch damping moment and the angular velocity is linear.

$$
N_{r y}=-C_{r y} \cdot r_{y}
$$

The initial length of the cable (the length without deformation under the initial equilibrium state) $l_{0}$ is related to the cable preload.

$$
l_{0}=\frac{l_{t} \cdot E A}{E A+T_{0}}
$$

$l_{\mathrm{t}}$ is the total length of the cable calculated from the distance between the winch and the anchor point, $T_{0}$ is the preload of the cable. From Eq.8 to Eq.18, the relationship between cable preload and ship motion state can be obtained.

\subsection{Environmental load calculation}

You It can be seen from the yaw motion equation that the main external environmental loads of the system mainly include wind, wave and current. At present, there is no accurate formula to describe these loads. The flow of Hanjiang River can be obtained by model test or empirical formula. This paper belongs to the preliminary design stage, using empirical formula to calculate ${ }^{[8-10]}$.

At present, the general empirical calculation formula of wind load is as follows:

$$
\left\{\begin{array}{l}
F_{\mathrm{x} \_ \text {wind }}=\rho_{a} V_{w}^{2} C_{x w} A_{\mathrm{T}} \\
F_{\mathrm{y} \_ \text {wind }}=\rho_{a} V_{w}^{2} C_{y w} A_{\mathrm{L}} \\
M_{\mathrm{z} \_ \text {wind }}=\rho_{a} V_{w}^{2} C_{z w} A_{\mathrm{L}} L_{\mathrm{bp}}
\end{array}\right.
$$

In Eq.19, $V_{\mathrm{w}}$ is the average stable wind speed of $10 \mathrm{~m}$ above the water surface; $A_{\mathrm{T}}$ and $A_{\mathrm{L}}$ is divided into forward projection and side projection area; $L_{\mathrm{bp}}$ is the length between the two columns of the ship; $C_{\mathrm{xw}}, C_{\mathrm{yw}}$, $C_{\mathrm{zw}}$ is dimensionless coefficient, which is calculated by empirical formula.
In this paper, the low-frequency oscillation of a water surface leveling barge is studied, that is, the yaw in the horizontal plane is considered. Because the amplitude of the first-order wave force is small and will automatically return to the equilibrium position, only the second-order drift force is considered and the first-order wave force is ignored. The general empirical formula of the second order steady drift force is as follows:

$$
\left\{\begin{array}{l}
F_{\mathrm{x}_{-} \text {wave }}=\rho_{w} g L_{b p} C_{\mathrm{x} d} \cos ^{3}\left(\theta_{d}-\psi\right) \\
F_{\text {y_wave }}=\rho_{w} g L_{b p} C_{y d} \sin ^{3}\left(\theta_{d}-\psi\right) \\
M_{\text {z_wave }}=\rho_{w} g L_{b p} C_{z d} \sin ^{2}\left(\theta_{d}-\psi\right)
\end{array}\right.
$$

Coefficient $C_{\mathrm{xd}}, C_{\mathrm{yd}}, C_{\mathrm{zd}}$ is the constant wave drift coefficient, which can be calculated by the following formula:

$$
\left\{\begin{array}{l}
C_{x d}=\int_{0}^{\infty} S\left(\omega_{0}\right)\left[\frac{F_{x d}\left(\omega_{0}\right)}{0.5 \rho_{0} g a^{2}}\right] \mathrm{d} \omega_{0} \\
C_{y d}=\int_{0}^{\infty} S\left(\omega_{0}\right)\left[\frac{F_{y d}\left(\omega_{0}\right)}{0.5 \rho_{0} g a^{2}}\right] \mathrm{d} \omega_{0} \\
C_{z d}=\int_{0}^{\infty} S\left(\omega_{0}\right)\left[\frac{C_{z d}\left(\omega_{0}\right)}{0.5 \rho_{0} g L_{b p} a^{2}}\right] \mathrm{d} \omega_{0}
\end{array}\right.
$$

$\mathrm{S}\left(\omega_{0}\right)$ is the spectral density coefficient of wave energy; $F_{\mathrm{xd}}\left(\omega_{0}\right), F_{\mathrm{yd}}\left(\omega_{0}\right), F_{\mathrm{zd}}\left(\omega_{0}\right)$ is the second-order wave force at frequency and $a$ is the amplitude.

For flow load, the following empirical formula can be used to calculate:

$$
\left\{\begin{array}{l}
F_{\mathrm{x} \__{-} \text {curr }}=\frac{1}{2} \rho_{w} V_{c u r}^{2} C_{x c} L_{\mathrm{bp}} d \\
F_{\mathrm{y} \_ \text {curr }}=\frac{1}{2} \rho_{w} V_{c u r}^{2} C_{y c} L_{\mathrm{bp}} d \\
M_{\mathrm{z} \__{-} \text {curr }}=\frac{1}{2} \rho_{w} V_{c u r}^{2} C_{z c} L_{\mathrm{bp}}^{2} d
\end{array}\right.
$$

\section{Controller design}

In the process of anchoring and positioning of riprap leveling ship, there are external factors such as wind, wave, current and the change of the position of the ship's center of gravity, so it is difficult to establish an accurate mathematical model of cable length. Therefore, a realtime feedback convergence control method of position information is adopted to control the length of cable. The basic principle is to use GPS to measure the ship's current position and target position coordinates, get the cable tension through the balance equation of six degrees of freedom, and then compare and process the cable length, output the winch signal, send and receive the specified rope length of the cable. After multiple shifts, the position deviation gradually converges, so as to realize the automatic displacement function of the leveling ship, as shown in Figure 4. After the ship moves to the predetermined position, the preload is applied, and then the real-time fine adjustment is carried out according to the balance equation. 


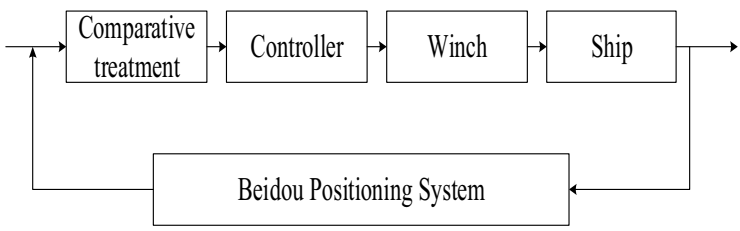

Fig. 4. Displacement control system of leveling ship

The cable length calculated by GPS is the original length of the cable, while the controller control and encoder feedback is the cable length without deformation. Therefore, it is necessary to measure the cable tension in real time through the tension sensor, and then calculate the deformation length of the cable, so as to obtain the non deformation length of the cable. The controller controls the action and speed of the winch.

There are three modes of winch retracting and releasing cable speed: high, medium and low. According to the position deviation, the displacement control of leveling ship can be divided into two stages: coarse displacement and fine adjustment. In the coarse displacement stage, the position deviation is large, and the medium high speed is used to realize the fast ship moving to the target area. In the stage of fine positioning, the position deviation is small, and the low speed control is used to realize the approximation.

The effective riprap operation area of the leveling ship at a certain position is about $46 \mathrm{~m} \times 13 \mathrm{~m}$, so the distance to be moved each time is $13 \mathrm{~m}$. This distance is converted into the cable car's retracting and releasing length. According to the required control time, the coarse control time and the fine control time are allocated to obtain the winch's retracting and releasing speed. The judgment basis of rough positioning and fine positioning and winch cable retraction speed are shown in Table 1.

Table 1. Difference between rough control and fine control

\begin{tabular}{|c|c|c|}
\hline & $\begin{array}{c}\text { Winch } \\
\text { retraction speed }\end{array}$ & $\begin{array}{c}\text { Position } \\
\text { deviation }\end{array}$ \\
\hline $\begin{array}{c}\text { Rough } \\
\text { control }\end{array}$ & $1.2 \mathrm{~m} / \mathrm{s} \sim 2.4 \mathrm{~m} / \mathrm{s}$ & $>1 \mathrm{~m}$ \\
\hline $\begin{array}{c}\text { Fine } \\
\text { control }\end{array}$ & $<0.4 \mathrm{~m} / \mathrm{s}$ & $<1 \mathrm{~m}$ \\
\hline
\end{tabular}

The control speed of the rope length is calculated according to the time requirement according to the maximum change of the rope length, and the speed of the other three winches is obtained in proportion.

$$
V_{\mathrm{i}}=\eta \cdot V \cdot \frac{\Delta l_{i}}{\Delta l_{\max }}(i=1,2,3,4)
$$

\section{Simulation and result analysis}

According to the established mathematical model, the simulation model is established. When the environmental forces along the $\mathrm{x}$-axis are $9800 \mathrm{n}$ and $19600 \mathrm{n}$ step forces of $5 \mathrm{~S}$, the simulation results are shown in Table 2.
Table 2. Simulation results of the influence of preload on ship stability

\begin{tabular}{|c|c|c|c|}
\hline $\begin{array}{c}\text { Environme } \\
\text { ntal forces } \\
(\mathrm{N})\end{array}$ & $\begin{array}{c}\text { Preload } \\
(\mathrm{N})\end{array}$ & $\begin{array}{c}\text { Maxdisplac } \\
\text { ement of x- } \\
\text { axis (m) }\end{array}$ & $\begin{array}{c}\text { Maximum pitch } \\
\text { angle (×E-4rad) }\end{array}$ \\
\hline 9800 & 9800 & 0.0989 & 1.081 \\
\hline 9800 & 29400 & 0.0985 & 1.077 \\
\hline 9800 & 49000 & 0.0981 & 1.072 \\
\hline 19600 & 9800 & 0.1960 & 2.134 \\
\hline 19600 & 29400 & 0.1951 & 1.125 \\
\hline 19600 & 49000 & 0.1943 & 2.115 \\
\hline
\end{tabular}

The simulation results of $9800 \mathrm{~N}$ and $49000 \mathrm{~N}$ are shown in Fig.5, Fig.6 and Fig.7. It can be seen from Fig.5 and Fig.6 that the vibration attenuation occurs under the environmental force, with the maximum displacement of $0.0981 \mathrm{~m}$ and the maximum pitch angle of $1.072 \mathrm{e}-4 \mathrm{rad}$. After 10 minutes, the displacement is about $0.0138 \mathrm{~m}$, and the pitch angle is about $1.54 \mathrm{e}-5 \mathrm{rad}$. It can be seen from figure 8 that due to the change of the leveling ship's attitude, the length of the cable is changed, and then the cable tension will attenuate vibration, and the tension of the four cables will eventually return to $49000 \mathrm{~N}$ (preload value). And in this process, the tension of each cable is always greater than 0 .

As shown in Fig.8, when the environmental force is about $62000 \mathrm{~N}$, the cable tension begins to appear negative, which means that the cable can no longer maintain tension.

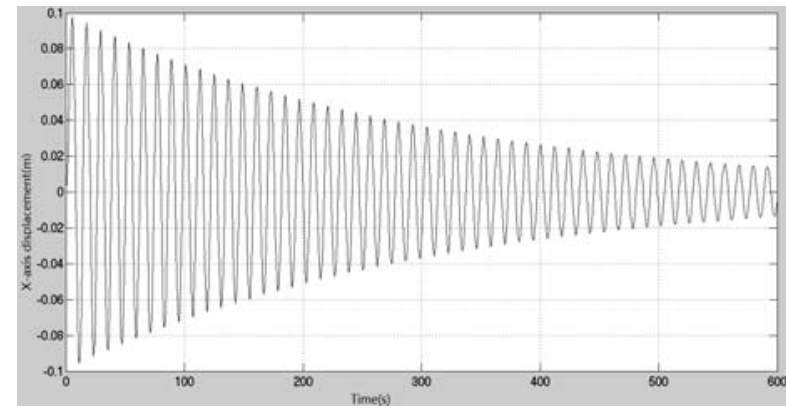

Fig. 5. The change trend of $x$-axis displacement when preload is $49000 \mathrm{~N}$.

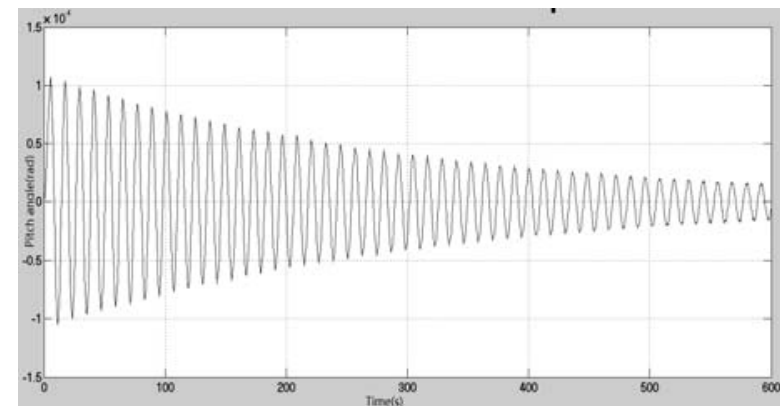

Fig. 6. Pitch angle change trend when preload is 49000 N. 


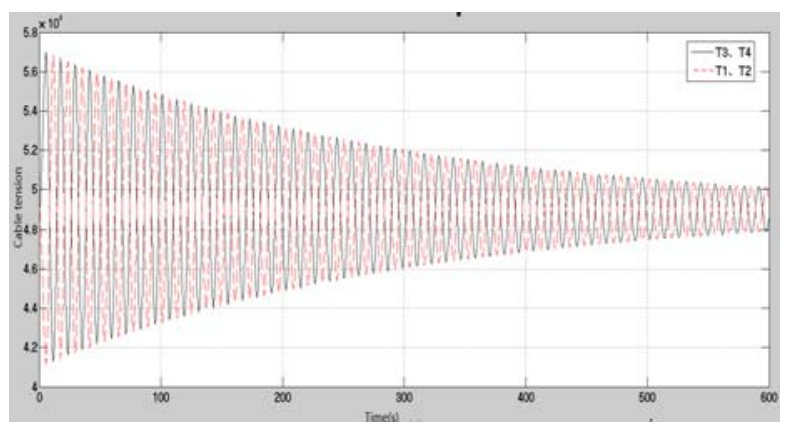

Fig. 7. Tension variation trend of four cables when preload is $49000 \mathrm{~N}$.

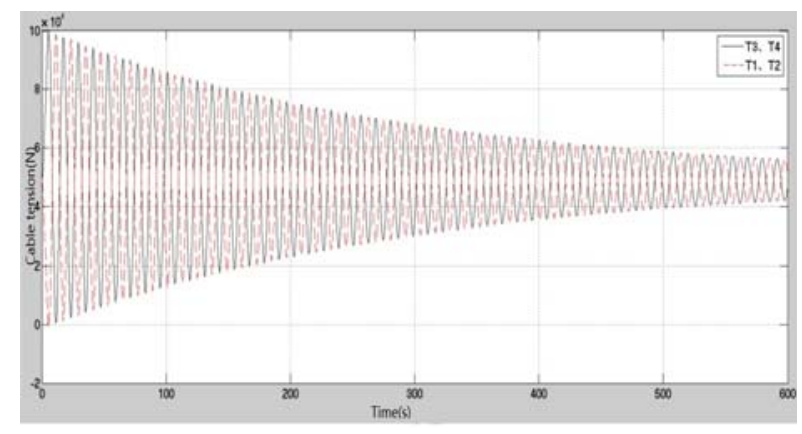

Fig. 8. When the environmental force is 62000 n and the preload is $49000 \mathrm{n}$, the cable tension change trend.

\section{Conclusion}

This paper introduces the composition of control system and construction process of leveling ship. Then, the six degree of freedom motion model of the leveling ship is established. The deformation of the cable is taken into account, which improves the control accuracy and meets the control requirements of the system. Based on the construction characteristics, a real-time feedback control system based on the balance equation of six degrees of freedom is designed. Pre tightening force is an important factor in the process of mooring and positioning of leveling ship. This paper analyzes the influence of cable preload on ship stability and carries out simulation. The simulation results show that, under the same environmental load conditions, the setting of preload has an impact on the stability of the ship. The larger the preload, the easier the ship is to maintain stability. When the pretightening force is constant, the position and angle deviation of the ship will increase with the increase of the environmental force. When the environmental force reaches a certain degree, some cables will relax, which is not conducive to the fixing of the leveling ship. Therefore, it is necessary to set the pre tightening force reasonably to avoid such phenomenon.

\section{References}

1. N. Kitney, D.T. Brown, J. Offshore Mech. Arct. Eng. Trans. ASME 123, 1-9 (2001)

2. R J. Smith, C J. MacFarlane, Ocean Eng. 28, 899914 (2001)
3. N Wang, XY Meng, QY Xu, ZW Wang, J. Navig. 64, 643-655(2009)

4. Z Pietrzykowski, J Uriasz, J. Navig. 62, 93108(2009)

5. T Perez, T I. Fossen, Ocean Eng. 38, 426-435(2011)

6. J Gobat, M Grosenbaugh, Ocean Res. 23, 159174(2001)

7. H.B Gu, D.M. Causon, C.G. Mingham, L.Qian, Eur. J. Mech. B-Fluids, 38, 1-17(2013)

8. D N. Daalisan, M T. Lim, Physica A, 457, 138147(2016)

9. E.A. Tannuri, H.M. Morrishita, Appl. Ocean Res. 28, 133-146(2006)

10. EW Bai, YF Huang, Automatica, 36, 10011008(2000) 